\title{
Lumière et gravitation : une préhistoire classique de la relativité générale
}

Jean Eisenstaedt (jean.eisenstaedt@obspm.fr)

Observatoire de Paris, SYRTE, 61 avenue de l'Observatoire, 75014 Paris

De la seconde moitié du $18^{\mathrm{e}}$

siècle au début du 19e siècle,

une optique relativiste classique

des corps en mouvement,

la théorie corpusculaire

de la propagation

de la lumière, application

parfaitement cohérente

des Principia d'Isaac Newton,

a été très précisément

développée.

Elle a en effet donné lieu

à un important corpus

de travaux qui constituent

un préambule aux relativités

d'Einstein, par la mise

en évidence de plusieurs

effets connus aujourd'hui

concernant l'interaction entre

la matière et les corpuscules

lumineux.

Ces calculs seront oubliés au

$19^{\mathrm{e}}$ siècle, en raison du choix

de la théorie ondulatoire.
Comment traitait-on de la propagation de la lumière après Newton, avant Einstein ? Dans un corpus du $18^{\mathrm{e}}$ siècle, ont été pensés, posés, calculés plusieurs effets qui, oubliés, ne seront vérifiés que bien plus tard. L'effet Doppler-Fizeau tout d'abord, mais aussi plusieurs effets des théories de la relativité d'Einstein : la déviation des rayons lumineux au voisinage d'un corps pesant, l'effet de décalage des raies, les corps obscurs, cousins des trous noirs, mais encore une expérience de pensée qui laisse entendre celle de Michelson.

Il s'agit bien d'une optique des corps en mouvement, la théorie newtonienne de la propagation de la lumière, une préhistoire classique aux relativités einsteiniennes. Une théorie corpusculaire que l'émergence de la théorie des ondulations de Fresnel fera oublier. Un oubli à peu près total, qui a persisté jusqu'à nos jours.

\section{La théorie corpusculaire de la lumière de Newton et les lois de la réfraction}

Dans les Principia de Newton, publiés en 1687, cinématique et dynamique des corps matériels sont pensées dans un cadre absolu et s'appuient sur le principe de relativité ainsi que sur la loi d'addition des vitesses de Galilée. Newton a accepté l'analyse de Römer selon laquelle la lumière a une vitesse finie et, dans le cadre de la Section XIV du Livre II des Principia, il développe quelques propositions où la lumière est traitée comme un corpuscule matériel. Il s'agit essentiellement d'une théorie de la réfraction, la théorie corpusculaire de la lumière. En fait, Newton ne se servira guère de ces

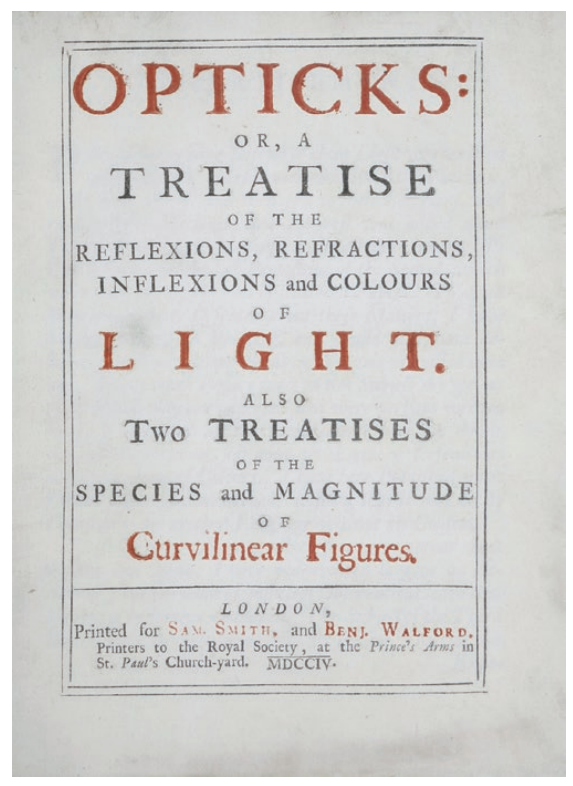

1. Couverture de la première édition en anglais de l'Optiks d'Isaac Newton, publiée en 1704.

quelques «propositions ", sinon pour retrouver la loi de Descartes et pour rendre compte de la réfraction astronomique (la déviation de la lumière due à la traversée de l'atmosphère); il vise l'étude du comportement de la lumière dans les verres. À la fin du $17^{\mathrm{e}}$ siècle, il tente de bâtir, sans succès, une théorie corpusculaire de la lumière où la vitesse aurait été le paramètre des couleurs, un sujet complexe qu'il reprendra, avec des idées bien différentes, dans son Opticks (fig. 1).

En 1741, Alexis-Claude Clairaut se penche sur l'optique corpusculaire qu'il expose algébriquement d'une manière plus claire que Newton, dont les propositions 


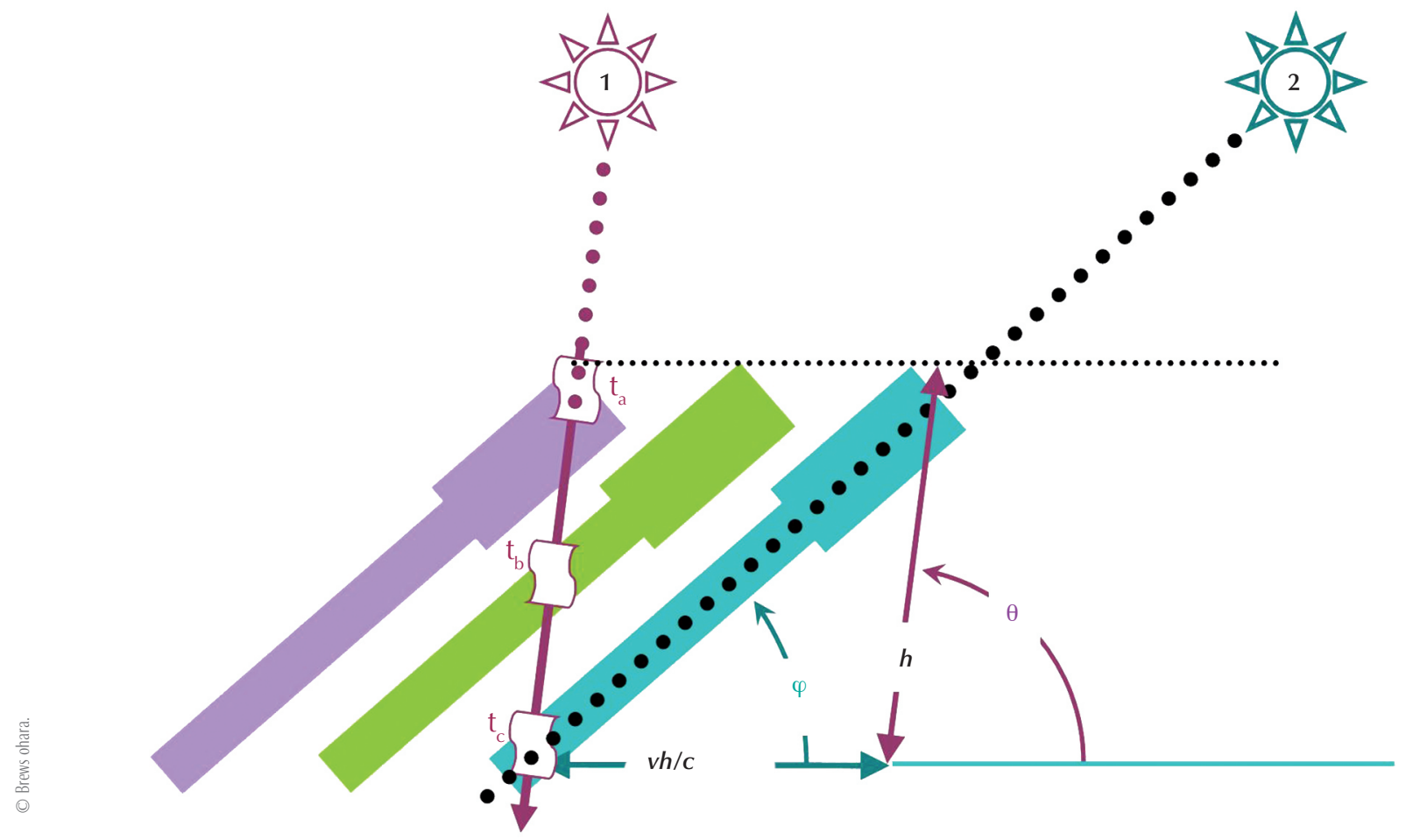

2. Explication classique de l'aberration des étoiles. Supposons qu'une étoile très éloignée est observée par un télescope (schématisé sur la figure par un tube). La lumière de l'étoile (rayons parallèles) arrive sous l'angle $\theta$ au temps $t_{a}$ à l'entrée du tube, et met un temps $h / c$ (c est la vitesse de la lumière) pour atteindre le bas du tube où elle est détectée au temps $t_{c}$. Les observations sont faites sur la Terre, qui se déplace avec une vitesse v. Durant le transit de la lumière dans le tube (entre les temps $t_{a}$ et $t_{c}$ ), le tube se déplace d'une distance vh/c. Pour que la lumière puisse atteindre le fond du tube (l'oculaire du télescope), ce dernier doit être incliné à un angle $\varphi$ différent de $\theta$. La position apparente 2 de l'étoile diffère donc de sa position réelle 1. À mesure que le mouvement de la Terre sur son orbite autour du Soleil change de direction, $\varphi$ change avec l'époque de l'année où l'observation est faite. La valeur de l'aberration ne dépend donc pas de la position de l'étoile, et produit une déviation apparente dans le plan contenant l'étoile et la vitesse de la Terre, ce qui distingue nettement ce phénomène de celui de la parallaxe stellaire.

étaient exprimées géométriquement. Il s'agit d'une optique balistique, et il n'y a que peu de différence entre les équations du mouvement d'une balle de mousquet soumise à la gravitation terrestre et celles d'un "globule lumineux ", comme le nomme Clairaut, soumis à la force réfringente - la force responsable de la réfraction d'un cristal. Simplement, la force réfringente s'exerce à courte portée sur les globules, tandis que la force de gravitation s'exerce sur tous les corps, essentiellement à longue portée.

\section{Le prisme, un outil pour mesurer la vitesse de la lumière}

L'optique corpusculaire de Newton permet de rendre compte de la réfraction d'un corpuscule lumineux réfracté par une lame, par un prisme. Le corpuscule incident, soumis à la force réfringente présente à la surface du verre, est infléchi. On en déduit la loi des sinus, de SnellDescartes, ainsi qu'une loi de force. Les sinus des angles d'incidence et de réfraction sont dans un rapport constant, inverse à celui des vitesses, qui n'est autre que l'indice de réfraction du verre.
Ainsi, l'angle d'incidence du corpuscule lumineux étant fixé, plus la vitesse d'incidence du corpuscule lumineux est grande, et moins l'angle de réfraction sera important; tout simplement parce que les sinus des angles d'incidence et de réfraction sont inversement proportionnels aux vitesses correspondantes. Cela signifie que, sur une lame de verre, à incidence constante, la mesure de l'angle de réfraction est une mesure de la vitesse du corpuscule.

Ainsi, un prisme est un outil pour mesurer une variation de la vitesse de la lumière, ce que comprendra John Michell dont l'article de 1784 - essentiel - est à l'origine de la plupart des travaux sur ce thème.

\section{L'aberration et la constance de la vitesse de la lumière}

En 1728, un an après la mort de Newton, James Bradley (1693-1762), alors astronome royal, découvre l'aberration (fig. 2), mouvement apparent des étoiles qui provient de la composition de la vitesse de la lumière, $c$, avec celle de la Terre, v. Pour une étoile au pôle, l'ellipse d'aberration dessine celle du mouvement de la Terre autour du Soleil. Mais les observations montrent que l'angle sous lequel on voit le grandaxe de cette ellipse, proportionnel à v/c, est constant. La vitesse de la Terre, v, étant pratiquement constante, cela signifie que $c$, la vitesse de la lumière, est constante pour toutes les étoiles.

$\mathrm{Au}$ début du $19^{\mathrm{e}}$ siècle, l'astronome Jean-Baptiste Delambre évalue la vitesse de la lumière grâce à la méthode de Römer, c'est-à-dire par la variation de la période des satellites de Jupiter en fonction du mouvement de la Terre sur son orbite, et trouve ainsi précisément le même résultat que celui qu'il pouvait tirer des données de Bradley.

Mais comment concilier la constance de la vitesse de la lumière et l'addition des vitesses, qui exige que l'on compose les vitesses d'émission de la source et de l'observateur ? Cette contradiction ne s'exprimera pas avant les travaux de François Arago, de 1806 à 1810, un point qui le convaincra de soutenir les travaux d'Augustin Fresnel. 
C'est pour mesurer la distance des étoiles qu'en 1784, le Révérend John Michell, un astronome anglais extrêmement profond, newtonien convaincu, ami de Henry Cavendish, s'appuyant sur la théorie corpusculaire de Newton, comprend qu'un prisme est un instrument qui devrait permettre sinon de mesurer la vitesse de la lumière, du moins d'en mesurer la variation. Michell pense ainsi pouvoir comparer les vitesses des rayons lumineux provenant de sources diverses.

\section{La lumière, sensible à la gravitation}

L'universalité de la gravitation étant pour Michell une évidence, il s'ensuit que la lumière doit, comme tout autre corpuscule, y être soumise. Un corpuscule lumineux émis par une étoile avec une vitesse constante sera petit à petit freiné, sa vitesse diminuée. À tel point que, si l'étoile est très massive, le corpuscule, tel une pierre jetée en l'air, peut s'arrêter dans sa course et retomber sur l'étoile. Ainsi invente-t-il ces objets étranges que Pierre-Simon Laplace nommera " corps obscurs " et qui s'apparentent aux trous noirs, fruits tardifs de la relativité générale.

En 1801, s'appuyant sur les travaux de Michell vulgarisés par Laplace, Georg von Soldner en déduira qu'un rayon lumineux peut être dévié de sa course s'il passe près d'un corps pesant. Ses résultats ne sont aucunement différents de ceux d'Einstein qui en 1911, songeant à observer ce phénomène lors d'une éclipse, calcule lui aussi la déviation dans le cadre newtonien. Après 1915, dans le cadre de la relativité générale, la déviation aura une valeur double.

Dans ce même article, extraordinairement innovant, Michell songe à une expérience très sophistiquée. Depuis 1767, il s'est rendu compte, grâce à des comptages liés à une analyse statistique, que les étoiles doubles sont particulièrement nombreuses, à tel point qu'il est persuadé de l'existence d'étoiles doubles physiques, gravitationnellement liées qui seront observées quelques années plus tard par William Herschel. Michell espère que l'on pourrait observer un système double dont l'étoile centrale serait très massive ; ainsi, la vitesse de la lumière émise en serait assez fortement diminuée (d'un vingtième, précise-t-il) tandis que celle de son compagnon, beaucoup moins massive, ne le serait que fort peu. Il espère que l'on pourra distinguer les deux faisceaux provenant d'un tel système double et parvenir à en mesurer (sur un prisme) les angles de réfraction. Ainsi devrait-on mesurer la différence entre des angles de réfraction des deux faisceaux, en déduire la différence de vitesses des corpuscules lumineux les composant, et en induire la masse de l'étoile. On ne s'étonnera pas que les meilleurs astronomes du temps, Nevil Maskelyne (Royal Astronomer) et Willam Herschel n'aient rien pu observer de tel. Mais ce nouveau phénomène a, lui aussi, quelque chose à voir avec la relativité générale car il s'agit précisément des prémices de ce que l'on nomme aujourd'hui le décalage gravitationnel des raies, "l'effet Einstein ».

\section{Mesurer la vitesse des étoiles, Blair invente l'effet Doppler-Fizeau}

Michell n'a pas pensé à appliquer sa " méthode " à des questions de cinématique. Mais deux ans plus tard, en 1786, Robert Blair (fig. 3), un astronome écossais fort peu apprécié de ses collègues et quasiment inconnu aujourd'hui, voit tout l'intérêt de la "méthode " de Michell. Il a fort bien compris que le prisme est un instrument pour mesurer des différences de vitesses de la lumière. Ainsi, il invente l'effet Doppler près de soixante ans avant celui-ci, et propose de très nombreuses observations. Son travail fut exposé le 6 avril 1786 devant la Royal Society et son manuscrit circula dans le milieu savant ; il ne fut jamais publié. Il est extrêmement proche de l'article dans lequel Armand-Hippolyte Fizeau théorisera à nouveau l'effet beaucoup plus clairement que Christian Doppler - en 1848. Ceci dit, l'effet Doppler-Fizeau prévoit un décalage des raies consécutif à une modification de leur fréquence, tandis que Blair ne peut penser qu'en terme de déviation. Car à la fin du $18^{\mathrm{e}}$ siècle, les raies ne sont ni bien observées ni comprises et le concept de fréquence n'est pas utilisé dans le cadre de la théorie corpusculaire. Mais il s'agit du même phénomène vu dans deux théories différentes, corpusculaire et ondulatoire. Fizeau fera le lien entre ces deux manières de prédire les décalages des raies, en 1870 .

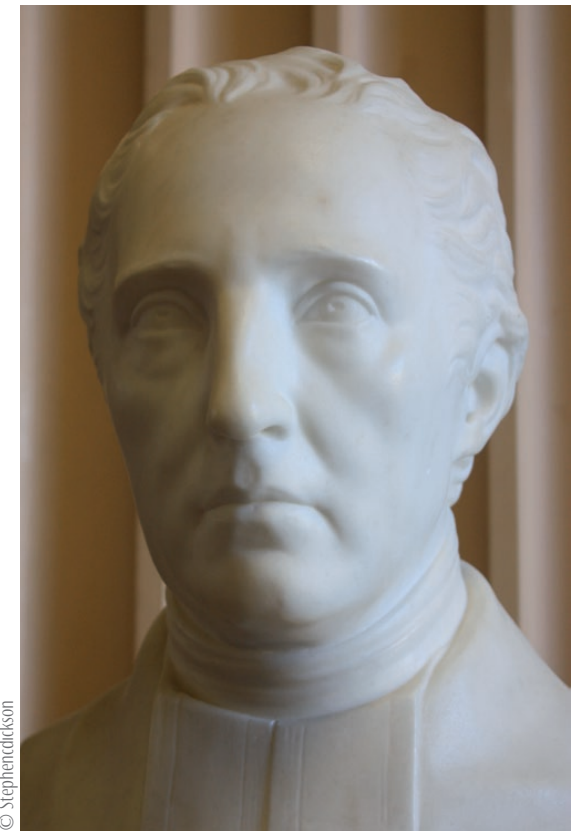

3. Buste de Robert Blair (1748-1828) par Thomas Campbell, 1815, Old College, Université d'Édimbourg.

Dans le manuscrit de Blair, ce phénomène est clairement explicité, et d'abord bien sûr la logique de la "méthode » de Michell, dont il souhaite avant tout vérifier la justesse. Bien que les calculs n'y soient pas développés, l'analyse de l'article permet de s'assurer que Blair les a correctement réalisés dans la logique de la théorie corpusculaire. Surtout, il a exprimé avec précision toutes les conséquences que l'on peut en tirer:

"Le mouvement de l'observateur étant donné, il s'agit de déterminer ainsi par un examen minutieux, le mouvement par lequel une quelconque planète, comète ou étoile fixe, aussi éloignée soit-elle, s'approche ou s'éloigne de l'observateur ; inversement, le mouvement $d u$ corps par lequel la lumière est émise ou réfléchie étant donné, déterminer la vitesse avec laquelle l'observateur s'approche ou s'éloigne de ce corps" (Blair, 1786).

Ainsi, non seulement pourra-t-on mesurer la vitesse du Soleil et la direction de son mouvement, mais encore la vitesse radiale des planètes, des comètes, des étoiles, et même leur vitesse de rotation ; c'est un véritable programme d'observation des vitesses radiales qu'il propose, tout à fait parallèle aux propositions que fera Fizeau au milieu du $19^{\mathrm{e}}$ siècle, mais qui ne seront réalisables que dans les années 1870.

Afin de faire ces mesures, Blair construit un instrument qui se révèle 
bien trop sophistiqué. La déviation attendue sur un seul prisme étant très faible et difficile à mesurer, il veut démultiplier l'effet en faisant passer le faisceau de corpuscules lumineux à travers une douzaine de prismes installés en série sur une sorte de roue. Comme Michell, il utilise des prismes achromatiques, chacun étant formé de plusieurs prismes accolés. L'utilisation de prismes achromatiques évite le manque de précision qu'engendraient les aberrations chromatiques dans les observations. Mais il écrase toutes les raies les unes sur les autres, ce qui alors ne semble pas problématique, car ces raies ne sont ni décrites ni comprises. Bref, la précision que l'on peut attendre d'un prisme achromatique est tout à fait insuffisante pour des mesures aussi délicates. Évidemment, la multiplication des prismes engendre une absorption extrêmement importante qui détruira ces efforts de précision. Blair n'observera rien, laissant à "quelqu'un de plus compétent "le soin de réaliser son programme.

\section{Blair entrevoit l'expérience de Michelson}

Comme Michell, Blair travaille dans la logique des Principia, mais il s'intéresse aussi à la théorie ondulatoire, dans laquelle il entrevoit, dans le cadre d'une expérience de pensée, un «bien curieux phénomène » auquel il ne croit d'ailleurs pas, pas plus qu'à la théorie qui le porte. La lumière étant, dans ce cadre, une ondulation de l'éther, sa vitesse ne dépend pas de celle de sa source, mais uniquement de celle de l'observateur. Se plaçant dans une pièce fermée, Blair imagine de mesurer, dans différentes directions, la vitesse de la lumière émise par une bougie. L'instrument participant du mouvement de la Terre, sa vitesse devrait être différente à l'est et à l'ouest, au nord et au sud de la bougie. L'angle de réfraction devrait donc être différent, de sorte que l'on pourrait déduire de ces mesures la vitesse de la Terre ainsi que sa direction... Il s'agit là d'une expérience de pensée, selon la logique même de l'expérience de Michelson qui, à la fin du $19^{\mathrm{e}}$ siècle, réalisera une expérience en quelque sorte parallèle mais d'une manière infiniment plus précise - grâce à des techniques interférométriques -, obtenant un résultat finalement négatif.

\section{Arago et la vitesse de la lumière}

Cette vision corpusculaire et " relativiste " - au sens propre - de la lumière n'est généralement pas explicitée ; sans doute parce que les observations liées à l'aberration laissent entendre que la vitesse de la lumière est, sinon constante, du moins indépendante de celle de sa source - ce qui pose un problème majeur. Mais cela reste un non-dit. C'est pourtant une question intéressante pour François Arago ; il réalisera vingt ans plus tard, différemment, l'expérience de Blair.

Au tout début du $19^{\mathrm{e}}$ siècle, Arago, tout jeune astronome, est un fervent adepte de Newton et, bien sûr, de la théorie corpusculaire. Il a une grande culture et, en particulier, il a lu très attentivement l'article de 1784 de Michell ; "la méthode de Michell » retient son attention et il s'en sert pour monter une série d'observations qui ont pour but de mesurer des différences de vitesses de la lumière en provenance d'étoiles diverses. Les raisons pour lesquelles la vitesse de la lumière issue de sources différentes, mesurée à divers moments ne doit pas être la même ne manquent pas, car la loi d'addition des vitesses implique non seulement la vitesse (supposée constante) d'émission de la lumière, mais aussi celles de la source et de l'observateur. Qui plus est, Arago prend à son compte les corps obscurs de Michell, ce qui pourrait impliquer une diminution formidable de la vitesse de la lumière, jusqu'à l'anéantir. Il y a donc là une contradiction : dans le contexte newtonien la vitesse de la lumière ne peut être constante, comme le laissent entendre les mesures.

Ainsi réalise-t-il à l'Observatoire de Paris, dans les années 1809-1810, une très longue série de mesures de l'angle de réfraction sur un prisme - toujours achromatique - de la lumière provenant de sources très diverses. L'instrument employé est plus simple que celui de Blair ; un seul prisme assujetti à une lunette; un quadrant permet des mesures précises, mais limitées par l'achromaticité du faisceau émergent. Le résultat de ses observations est négatif et Arago a bien du mal à le comprendre ; faut-il remettre en cause la théorie corpusculaire ? Tel sera finalement son point de vue, puisqu'il encourage et soutient bientôt Augustin Fresnel activement. L'expérience "négative " d'Arago aura une très grande influence sur l'optique du $19^{\mathrm{e}}$ siècle, une influence ambiguë. En effet, réalisée au tournant des années 1870 dans de bien meilleures conditions expérimentales, ce même type d'expérience permettra - dans le cadre de l'effet Blair-(Doppler)Fizeau - de mesurer les vitesses radiales des étoiles.

La théorie newtonienne de la propagation de la lumière ne fut jamais publiée en tant que telle, mais elle fut largement utilisée. Après Fresnel, ces travaux, ce corpus, désormais inutile, sera presque totalement oublié. L'optique des corps en mouvement dans le contexte newtonien est pourtant un préambule naturel à la cinématique relativiste d'Einstein, un raccourci saisissant qui permet de mieux comprendre la raison de la physique des relativités.

\section{Références}

Dans ce court article il ne m’a pas été possible de préciser bien des points, bien des développements que l'on pourra trouver, ainsi que les références à la littérature correspondante, dans les travaux suivants

J. Eisenstaedt, "Light and relativity, a previously unknown eighteenth-century manuscript by Robert Blair (1748-1828)", Annals of Science 62 (2005) 347-376. [L'invention, bien avant Doppler et bien mieux que lui, de l'effet qui malencontreusement porte le nom de ce dernier.]

J. Eisenstaedt, Avant Einstein : relativité, lumière, gravitation, Seuil, Paris (2005). [0ù l'on trouvera outre les développements de cette histoire, les références des travaux originaux aussi bien que ceux, très mal connus, de la littérature secondaire.]

J. Eisenstaedt, « De Newton à Einstein : l'optique oubliée des corps en mouvement ", Bulletin de l'Union des Professeurs de Physique, 102 (2008) 1353-1368.

[Traduction: Reprinted with permission from J. Eisenstaedt, American Journal of Physics, 75 (2007) 741-746.] [0ù l'on trouvera l'ensemble des équations de la théorie newtonienne de la propagation de la lumière.]

J. Eisenstaedt et M. Combes, « Arago et la vitesse de la lumière (1806-1810), un manuscrit inédit, une nouvelle analyse ", Revue d'histoire des Sciences 64 (2011) 59-120. [Un supplément à Avant Einstein, où l'on retrouve le brouillon de l'article d'Arago (1806) et sa dérivation des équations du mouvement.]

R. McCormmach, Weighing the World: the Reverend John Michell of Thornhill, Springer (2012).

[Un splendide ouvrage consacré à Michell par l'un des très rares historiens à avoir travaillé sur cette histoire.] 